Case Report

\title{
MECKEL-GRUBER SYNDROME - A CASE REPORT
}

\author{
Naveen N.S. ${ }^{1}$, Vishal K. ${ }^{2} \&$ Vinay K.V. ${ }^{3}$ \\ ${ }^{1}$ Department of Anatomy, Raichur Institute of Medical Science, Raichur, Karnataka, India. \\ ${ }^{2,3}$ Department of Anatomy K.S. Hegde Medical Academy, Nitte University, Deralakatte, Mangalore, Karnataka. India. \\ Correspondence: \\ Vishal Kumar, \\ Associate Professor, Department of Anatomy, \\ K.S. Hegde M edical Academy, Nitte University, Deralakatte, M angalore - 575 018, Karnataka, India. \\ Mobile : +9198453 58754, E-mail vishalkumarmd@gmail.com.
}

\section{Abstract:}

Meckel Gruber syndrome (MGS) is a rare lethal autosomal recessive disorder. It is characterized by triad of features having occipital meningoencephalocoele, polycystic kidneys and post-axial polydactyly. We report a rare case of M GS which was diagnosed by antenatal ultrasound examination and confirmed later when aborted at 21 weeks of gestation.

Keywords: M eningoencephalocoele; Polydactyly; Polycystic kidney

\section{Introduction:}

Meckel Gruber syndrome (MGS) was first described by Johann Friedrick Meckel in 1822. It is also called as dysencephalia splanchnocystica or $\mathrm{MKS}^{1,2}$. It is a lethal autosomal recessive disease with gene locus mapped to chromosome 17q2 1-q24 ${ }^{2}$. The MGS is characterized by triad of features having occipital encephalocele, cystic kidneys and polydactyly ${ }^{3,45,6,7,78}$. It has wide phenotypic variations showing abnormalities of lip, palate, eye, ductal plates of the liver, cardiovascular and genital systems ${ }^{3,5,7,8}$. Other variants described include M KS2 in the Middle East and Northern African families, and recently M KS3 ${ }^{1}$. A high incidence of M KS has been reported in Gujarati Indians ${ }^{3}$. We report on a MGS diagnosed in the $2^{\text {nd }}$ trimester antenatal ultrasonogram examination and subsequently confirmed in the abortus for its rarity.

\section{Case Report:}

A routine antenatal sonogram was done on a 23 year old primi gravida mother at 22 weeks of gestation with the

\begin{tabular}{|c|}
\hline Access this article online \\
\hline Quick Response Code \\
\hline
\end{tabular}
history of third degree consanguineous marriage. The sonogram revealed an occipital encephalocele ( F i g. N 0.1) a nd hyperechoic enlarged kidneys (Fig. No.2). The occipital bone of the skull was deficient with out-pouching of the contents of the brain suggestive of occipital meningoencephalocele (Fig. No.3). The abdomen was distended (Fig. No.4). Post axial polydactyly with presence of sixth digit was noted in both lower limbs (Fig. No.5). There was hypertelorism (Fig. No.3). The foetus weighing about 430 grams was terminated. The detailed examination of the aborted fetus could not be done since the autopsy was refused by the parents.

\section{Discussion :}

M GS is prevalent worldwide, with equal male and female sex ratio ${ }^{2,5,6}$. The incidence worldwide has been reported as 1 in 13,250 to 1 in 1,40,000 live births ${ }^{1}$. The incidence of MGS in Gujarati Indians settled in Leicestershire is as high as 1:1300. This could probably be due to high mutation rate, heterozygote advantage or heterogeneity ${ }^{3}$.

MGS involves multiple organ systems of the body. The central nervous system (CNS) malformation ranges from total craniorachischisis to a partial defect of the corpus callosum. In CNS the most typical malformation is microcephaly with a sloping forehead and occipital meningoencephalocele. The occipital encephalocele results from an apical defect of the occipital bone or enlarged posterior fontanelle ${ }^{1,2}$. The occipital encephalocele was also observed in the present case. The abnormalities of the head and neck such as anencephaly, 


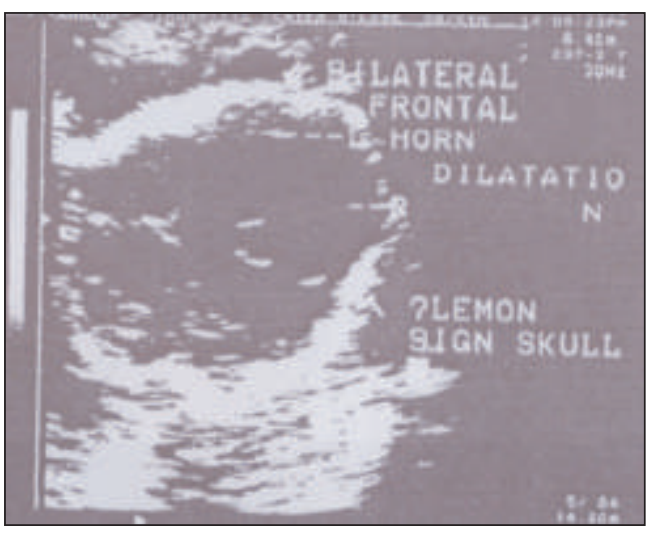

Figure 1. Ultrasonogram of fetus showing dilated frontal horns.

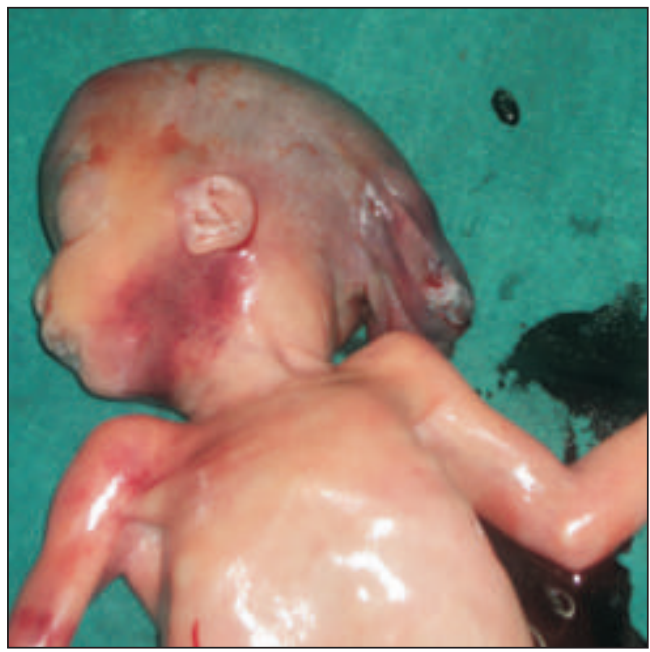

Figure 3. Fetus with deficient occipital bone with out-pouching of contents of brain.

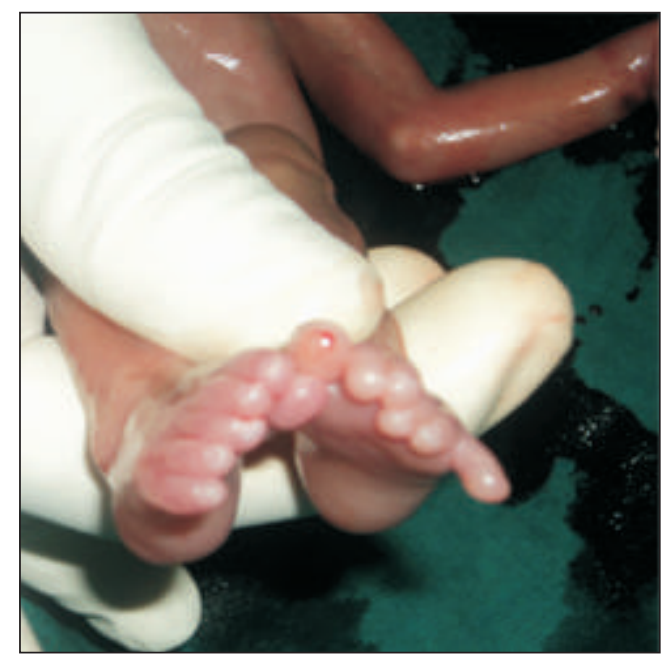

Figure 5. Fetus with sixth toe in both feet.

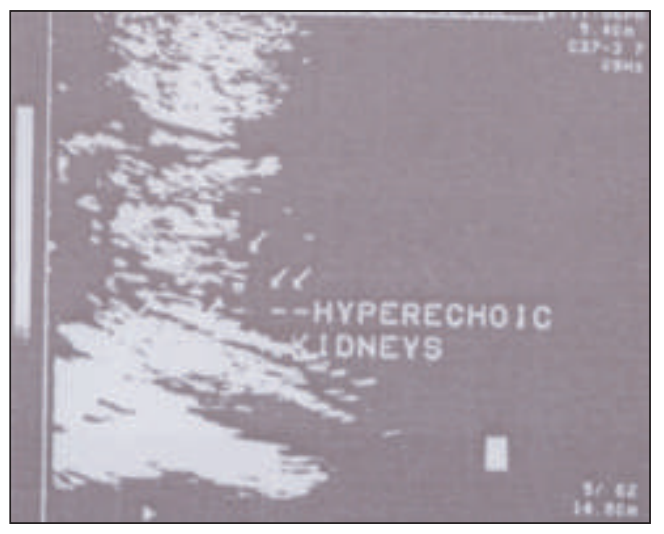

Figure 2. Ultrasonogram of fetus showing hyperechoic kidneys.

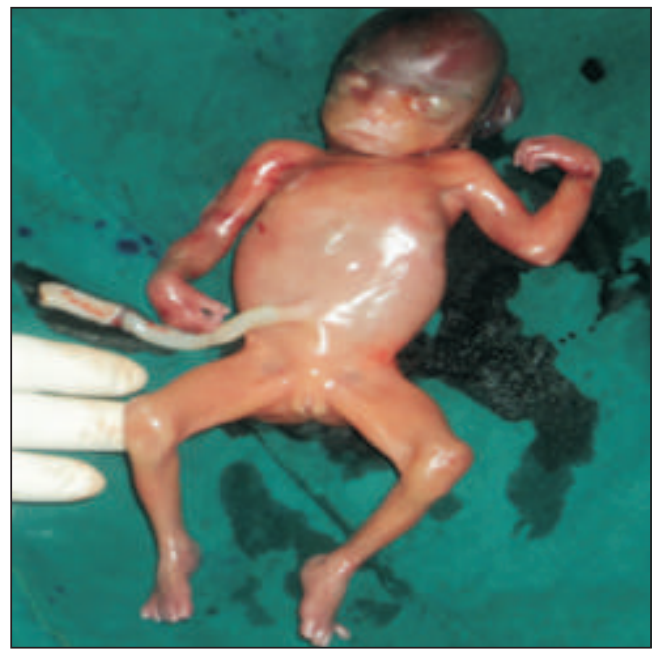

Figure 4. Fetus with enlarged abdome and ocular hypertelorism.

cerebral or cerebellar hypoplasia, hydrocephalus with or without Arnold - Chiari malformation, absence of olfactory tract or lobe, absence of Corpus Callosum and septum pellucidum may also be seen in this condition. Microphthalmia, cleft palate, micrognathia, ear abnormalities and short neck may also be seen ${ }^{1}$.

The kidneys are grossly enlarged by mid-trimester due to cystic changes ${ }^{1,2,6}$. Cystic dysplasia of the kidneys is the most constant characteristic feature of MGS. Due to this there is oligo-hydramnios and pulmonary hypoplasia which is the main cause of death. Kidneys may be enlarged 10-20 times the normal size. In the present case the enlarged and cystic changes in the kidneys were observed was in the sonogram at $22^{\text {nd }}$ week. Since the couple refused for autopsy, detailed study of kidneys couldn't be done. 
Postaxial polydactyly is a frequent finding in MGS ${ }^{2,6}$. Club foot is common because of oligo or anhydramnios ${ }^{6}$. In the present case, bilateral polydactyly with presence of sixth digit were seen in both the lower limbs.

Other organ involvement include intrahepatic bile duct anomalies and fibrotic changes in the portal areas of the liver, duct dilatation and periductal fibrosis in pancreas, hypoplastic or ambiguous genitalia, congenital heart defects, cleft lip and palate. Malformations like cleft lip, cleft palate, polydactyly, syndactyly and congenital heart defect occur in relatives of MGS more frequently than expected $^{6}$.

Prenatal diagnosis of MGS can be done by vaginal ultrasound scan after 11-12 weeks of gestation and chorionic villi sampling at 14 weeks for molecular diagnosis. M RI can also be used to see foetal defects ${ }^{1}$. In the present case, $1^{\text {st }}$ trimester scan did not show any

\section{References:}

1. Ramachandran U, Malla T, Joshi KS. M eckel-Gruber syndrome - Case Note. Kathmandu University M edical Journal 2006; 4 (3), 15: 334-36.

2. Salonen R, Paavola P. M eckel syndrome. Journal of M edical Genetics 1998; 35: 497-501

3. Young I D, Rickett AB, Clarke M. High incidence of M eckel's syndrome in Gujarati Indians. Journal of M edical Genetics 1985; 22: 301-04

4. Wright C, Healicon R, English C, Burn J. M eckel syndrome: what are the minimum diagnostic criteria? Journal of Medical Genetics 1994; 31: 482-85.

5. Sudan MS, Gupta S, Sharma N, Nargotra RC. Meckel-Gruber Syndrome: Sonographic Detection- Case report. JK Science 1999;1(1): 30-32.

6. Fried K, Liban E, Lurie M, Friedman S, Reisner SH. Polycystic Kidneys Associated with Malformations of the Brain, Polydactyly and Other Birth defects in Newborn Sibs. Journal of M edical Genetics 1971; 8: 285-90.

7. Balci $S$ et al., Prenatal diagnosis of Meckel-Gruber syndrome and Dandy-Walker malformation in four consecutive affected siblings, with the fourth one being diagnosed prenatally at 22 weeks of gestation- A Case. The Turkish Journal of Pediatrics 2004; 46: 283-88.

8. Gupta M, M ehta A, Gupta R, Gupta R, Singh S. Prenatal Diagnosis of Meckel-Gruber Syndrome with Dandy Walker Malformation- Case report. JKScience 2005; 7(3): 164-66. abnormality. Since MGS generally results inutero or neonatal death within a few hours after delivery, prenatal diagnosis is very important ${ }^{5,8}$. Chromosomal studies should be done to exclude trisomy $13^{1,8}$. In trisomy 13 , microcephaly often with alobar holoprosencephaly may be seen with bilateral cleft lip and palate, which are not so common in MGS. Polydactyly is common in the hands but rare in the feet in trisomy 13. Cystic kidneys can occur in trisomy 13.

\section{Conclusion :}

MGS is very rarely encountered in clinical practice and diagnosed incidentally on antenatal ultrasound evaluation. A detailed family history, accurate prenatal diagnosis with ultrasonography and measurement of alpha-fetoprotein levels in aminotic fluid are essential in diagnosing M GS. In pregnancy complicated by MGS, counselling for abortion and evaluation of recurrence risk in the future pregnancies are important. 